\title{
Laparoscopic Pediatric Inguinal Hernia Repair; Intracorporeal Purse-String Suture Using Needlescopic 2-mm Instruments
}

\author{
Jeong Min Lim, M.D., Hye Kyung Chang, M.D., Ph.D., Sun Jin Park, M.D., Ph.D. \\ Department of Surgery, College of Medicine, Kyung Hee University, Seoul, Korea
}

Purpose: Two-millimeter needlescopic instruments induce minimal damage to the abdominal wall and have excellent cosmetic benefits. We aimed to evaluate the feasibility of a laparoscopic intracorporeal suture using 2-mm instruments for pediatric inguinal hernia.

Methods: We retrospectively reviewed 131 patients who underwent laparoscopic repair between March 2011 and February 2017. Three trocars were used: a 5-mm umbilical trocar for a needle holder and two 2-mm trocars for a camera and a grasper. The internal ring was closed with an intracorporeal purse-string suture. A telephone interview was conducted to confirm recurrence.

Results: In the 131 patients, 169 procedures were successfully performed. The ages ranged from 2 months to 14 years (mean, 52.5 months), and the mean body weight was $18.0 \mathrm{~kg}$ (range, $6.7 \sim 49 \mathrm{~kg}$ ). The mean operating time was 42 minutes for the unilateral cases and 46 minutes for the bilateral inguinal hernia repairs. All the cases were completed laparoscopically without intraoperative complications. Herniotomy was not performed in all the patients except nine. A contralateral patent processus vaginalis was present in $27.3 \%(35 / 128)$ of the patients. During the mean follow-up period of 54.6 months, 3 recurrences $(2.3 \%)$ were observed. Two recurrences were treated using laparoscopy and one using open herniorrhaphy. Hydrocele occurred in one male patient. No wound complications or umbilical hernias developed. No testicular atrophy was observed.

Conclusion: This study showed that laparoscopic intracorporeal internal ring suture using 2-mm instruments for pediatric inguinal hernia was technically feasible and safe, with excellent cosmetic results.

Keywords: Inguinal hernia, Pediatrics, Laparoscopy, Suture technique, Inguinal ring

This is an Open Access article distributed under the terms of the Creative Commons Attribution Non-Commercial License (http:// creativecommons.org/licenses/by-nc/4.0/) which permits unrestricted non-commercial use, distribution, and reproduction in any medium, provided the original work is properly cited.
Received August 29, 2019

Revised September 16, 2019

Accepted October 11, 2019

Corresponding author

Sun Jin Park

Department of Surgery, Kyung Hee University Hospital, 23 Kyungheedaero, Dongdaemun-gu, Seoul

02447, Korea

Tel: $+82-2-958-8241$

Fax: +82-2-966-9366

E-mail: gsdrpark@naver.com ORCID:

https://orcid.org/0000-0001-7117-4479
Copyright @ 2020 The Journal of Minimally Invasive Surgery. All rights reserved.

\section{INTRODUCTION}

Inguinal hernia is one of the most common diseases in children that require surgery. Open herniotomy with high ligation is still regarded as the gold standard for pediatric hernia treatment. ${ }^{1}$ In the current laparoscopic era, however, the use of laparoscopic surgery in pediatric inguinal hernia repair is gradually increasing. Recently, many studies on the feasibility and safety of various methods of laparoscopic repair have been conducted, and these methods have become alternative treatment modalities to open surgery. ${ }^{1-5}$ Laparoscopic hernia repair has obvious advantages, including cosmetically better outcomes and identification of the contralateral side. ${ }^{1,3,5,6} \mathrm{How}^{-}$ ever, current evidence is insufficient to support its widespread use in children, and concerns against laparoscopic repair have been raised, particularly about the increased incidence of recurrences, longer operating times, and higher cost. ${ }^{3}$

Suturing and closing the internal inguinal ring are the key 
procedures for laparoscopic hernia repair. The procedure has many variations described in the literature, ${ }^{8}$ categorized briefly into intracorporeal and extracorporeal techniques. Intracorporeal techniques refer to the use of laparoscopic instruments to suture the internal ring and tie the knot intraperitoneally while avoiding the injury of the vas deferens and vessels. The internal ring is intracorporeally stitched using various methods such as the purse-string, Z-type, W-type, or flip-flap suture technique. ${ }^{9-12}$ Such intracorporeal suture is a basic procedure in laparoscopic surgery but requires adequate experience for satisfactory performance. ${ }^{13}$ On the other hand, extracorporeal techniques involve suturing the inner ring with a specialized needle instrument inserted percutaneously, where the knot is subcutaneously located outside the fascia. ${ }^{8}$ We performed laparoscopic intracorporeal purse-string suture using a combination of one 5-mm and two 2-mm instruments for pediatric inguinal hernia repair. The aim of this study was to evaluate the feasibility of our laparoscopic approach using 2-mm needlescopic instruments in pediatric patients with inguinal hernias.

\section{MATERIALS AND METHODS}

We retrospectively reviewed the electronic medical records of all pediatric patients who underwent laparoscopic inguinal hernia repair between March 2011 and February 2017 in our hospital. All the procedures were performed by a single attending surgeon at Kyung Hee University Medical Center. The patient characteristics included age, sex, weight, hernia side, and the presence of contralateral patent processus vaginalis (CPPV). Clinical results regarding the operation time, length of hospital stay, postoperative complications, recurrence rate, incidence of metachronous contralateral hernia, and follow-up period were studied. All the patients were followed up through a telephone survey conducted in July 2019, and the follow-up period was set from the date of surgery to the day of response to the telephone survey. In total, 110 of the 131 patients responded to the telephone questionnaire (response rate: 83.9\%). For the patients who did not answer the telephone survey, the follow-up period was set to the last day they visited the hospital. This study was approved by the Kyung Hee University Medical Center institutional review board (IRB No. 2019-06054-003).

\section{Operative procedure}

Under general anesthesia, the patient was placed in the Trendelenburg position with a slight tilting to the contralateral side of the hernia. A 5-mm vertical infraumbilical incision was made for the placement of a 5-mm trocar, and then

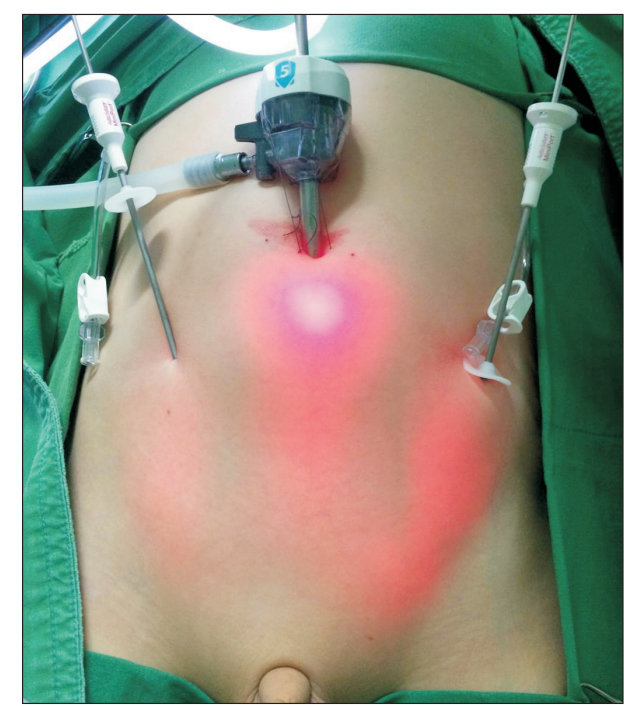

Fig. 1. The location of three trocars. The operator stood on the left side of the patient, holding a 5-mm laparoscopic holder with his right hand, and grasping a 2-mm grasper with his left hand, and a 2-mm camera located on the right side visualized the operating field.

a suture fixation was made to the skin to stabilize the trocar. Carbon dioxide pneumoperitoneum $(8 \sim 10 \mathrm{mmHg})$ was maintained during surgery. First, a 5-mm laparoscope was inserted through the 5-mm trocar, and the internal ring and presence of CPPV were assessed. Two 2-mm stab incisions were separately made for the $2-\mathrm{mm}$ trocar in the lateral side of both rectus muscles at the umbilical level (Fig. 1). If sliding contents were found in the hernia sac, they were cautiously pulled out using two 2-mm instruments before a purse-string suture was made. A 2-mm laparoscope was placed through the 2-mm trocar on the patient's right side. A 3-0 Prolene suture with a 17-mm needle (Ethicon Endo-Surgery, Blue Ash, OH, USA) prepared in a length of 10 to $12 \mathrm{~cm}$ and then was introduced in the abdominal cavity through a 5-mm trocar under the guidance of a 2-mm grasper. Then, a 5-mm needle holder was placed through the umbilical trocar and a 2-mm grasper, through the left trocar. Thus, the operator stood on the left side of the patient, holding a 5-mm laparoscopic holder with his right hand and grasping a 2-mm grasper with his left hand. A 2-mm camera located on the right side visualized the operating field. The internal ring was sewn with a pursestring suture while avoiding the visceral injury; the suture included enough subperitoneal tissue at the lateral circumference, whereas only the peritoneum at the lower circumference was included by carefully lifting the peritoneum with the help of a 2-mm grasper for the protection of the vas deferens and spermatic vessels (Fig. 2A). Before tying the knot, the scrotum or labia was squeezed to completely collapse the space of the 

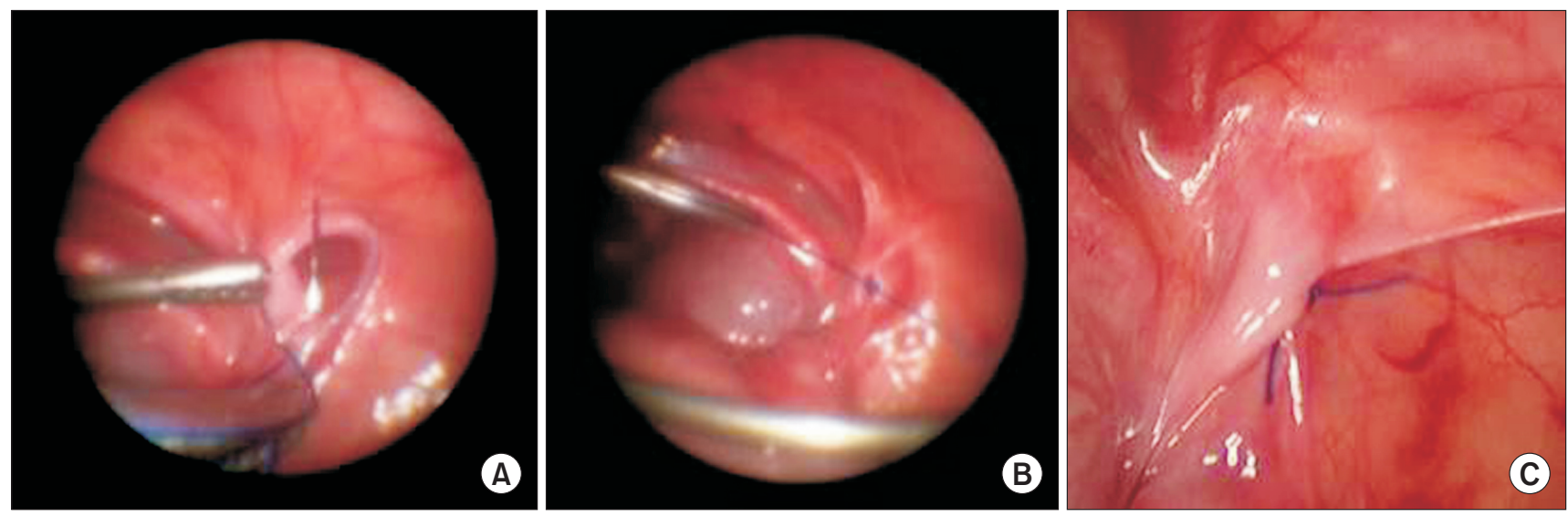

Fig. 2. Intracorporeal purse-string suture. The internal ring was sewn with a purse-string suture while avoiding the visceral injury (A). The 2-mm grasper held the thread when knotting (B, 2-mm camera view). The tie was completed to form an air tightening knot (C, 5-mm camera view).

hernia sac, and the tie was intracorporeally completed to form an air tightening knot (Fig. 2B, C). If a CPPV was found, it was closed in the same manner using a new 3-0 Prolene suture. Generally, herniotomy was not performed. The umbilical trocar wound was closed in 2 layers; the fascia was closed with a 3-0 Vicryl (Ethicon Endo-Surgery, Blue Ash, OH, USA), and the skin was closed with a subcuticular suture using a 4-0 Vicryl. The 2-mm trocar sites were approximated with skin tape. Oral intake was initiated 6 hours after general anesthesia. Patients without fever or other complications were discharged on the evening of surgery. All the patients were asked to make an outpatient visit 1 week after surgery for follow-up checkup for possible complications such as wound infection or hematoma.

\section{Statistical analysis}

Statistical analysis was performed using SPSS 23 version 23.0 (Statistical Package for Social Science, IBM Corporation, Chicago, IL, USA). For continuous variables, data were expressed as median or mean \pm standard deviation (SD). Categorical variables were expressed as frequency number and percentage.

\section{RESULTS}

During the study period, 169 laparoscopic inguinal hernia repairs were performed in 131 children. None of the patients had a conversion to open surgery. The patients' characteristics and clinical results are summarized in Table 1. The median total hospital stay was 1 day (range, 1 2 days), and the median postoperative hospital stay was 0 day (range, $0 \sim 1$ day). The mean follow-up period was 54.6 months (range, 0 99 months),
Table 1. Characteristics of patients and operative outcomes

\begin{tabular}{|lc|}
\hline \multicolumn{1}{|c|}{ Variable } & $\mathbf{n}(\%)$ \\
\hline Total number of patients & 131 \\
\hline Number of hernias repaired & 169 \\
\hline Male : female ratio & $85: 46$ \\
\hline Age (month) & $52.5(2 \sim 160)$ \\
\hline Weight (kg) & $18(6.7 \sim 49)$ \\
\hline Presentation & \\
\hline Symptomatic bilateral hernia & $3(2.2)$ \\
\hline Asymptomatic CPPV & $35 / 128(27.3)$ \\
\hline Metachronous hernia & $3(2.3)$ \\
\hline Recurrence & \\
\hline For patient & $3 / 131(2.3)$ \\
\hline For procedure & $3 / 169(1.8)$ \\
\hline For symptomatic hernia & $3 / 134(2.2)$ \\
\hline Operation time (minutes, mean \pm SD) & \\
\hline Unilateral repair & $42.39 \pm 14.04$ \\
\hline Bilateral repair & $46.45 \pm 12.24$ \\
\hline Total hospital stay (days) & $1(1 \sim 2)$ \\
\hline Postoperative hospital stay (days) & $0(0 \sim 1)$ \\
\hline Complications & $2 / 87(2.3)$ \\
\hline Scrotal swelling & $1 / 87(1.1)$ \\
\hline Hydrocele, postoperative & $54.6(0 \sim 99)$ \\
\hline Follow-up period (months, range) & \\
\hline
\end{tabular}

$\mathrm{CPPV}=$ contralateral patent processus vaginalis; $\mathrm{SD}=$ standard deviation . 
and the ratio of boys to girls was $85: 46$. The patients' mean age was 52.5 months (range, 2 160 months) and mean body weight was $18.0 \mathrm{~kg}$ (range, 6.7 49 kg). Among the 131 patients, 3 (2.3\%) had symptomatic bilateral inguinal hernia at diagnosis. Among the 128 patients with unilateral hernia, 35 (27.3\%) had a CPPV. Ninety-three unilateral hernias and 38 bilateral hernias were included. Herniotomy was not performed in all the patients except nine. Of the 85 male patients, 21 (25\%) had an accompanying hydrocele. Among the 93 unilateral hernias, 4 were metachronous contralateral inguinal hernias (4.3\%). Recurrence developed in 3 of the 134 symptomatic hernias (2.2\%, 2 boys and 1 girl) and in $1.8 \%$ of the 169 hernia operations. One 14-month-old boy, who was born with a preterm age of 31 weeks and a very low birth weight of 1,224 g, had a huge hernia since birth. He had recurrence 2 months after surgery and was corrected with open herniorrhaphy. Another 33-month-old boy and one 7-year-old girl had recurrence 2 months and 5 months after surgery, respectively, and were corrected by laparoscopy again. In the reoperation, the previous suture knot was found in the middle of the recurrent hernia sac in the former 1 case and was at the orifice of the

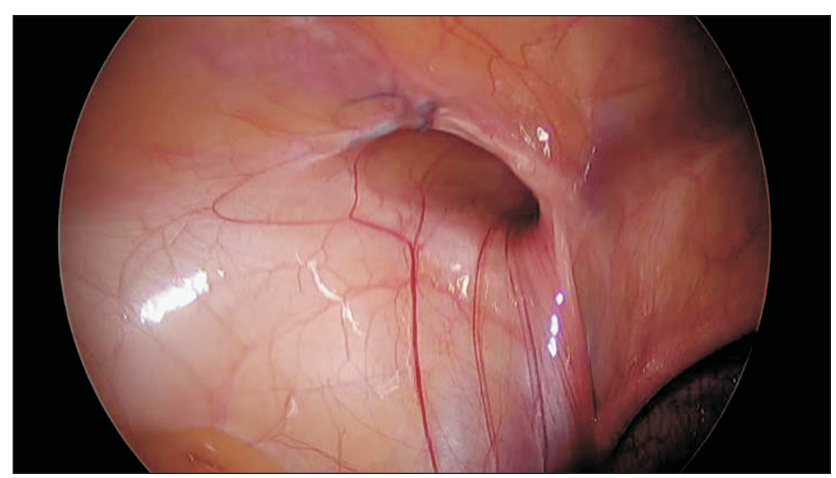

Fig. 3. Recurrent left inguinal hernia in a girl. The former suture knot was found at the orifice of the re-canalized internal ring.

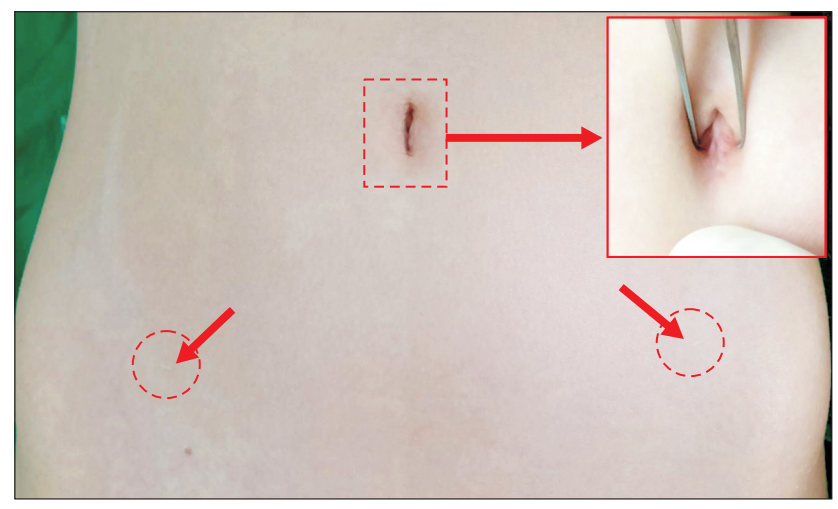

Fig. 4. Postoperative scar. recanalized internal ring in the latter 2 cases, the upper and lateral sides of the orifice (Fig. 3). Postoperative hydrocele occurred in 1 male patient, which was not resolved spontaneously after 3 months and was corrected with laparoscopic repair. The operating time from the incision to the skin closure was $42.39 \pm 14.04$ minutes for the unilateral hernia repair and 46.45 \pm 12.24 minutes for the bilateral repair. Postoperative scrotal swelling was observed in 2 patients but disappeared spontaneously. No wound infection or umbilical hernia was observed. No testicular atrophy was observed.

\section{DISCUSSION}

This study supports that the combined use of two 2-mm needlescopic instruments and one $5-\mathrm{mm}$ instrument is feasible in laparoscopic intracorporeal suture for pediatric inguinal hernia repair. Although this study did not investigate patient postoperative pain or satisfaction, the small incisions clearly left small scars (Fig. 4). The 2-mm needlescopic instruments cause minimal tissue damage and have excellent cosmetic results, but they are not easy to handle because their jaws are weaker and smaller than those of 5-mm instruments. In our experience, needles are not easy to hold and control with 2-mm instruments. Thus, in the instrument setting, a 5-mm needle holder was placed in the $5-\mathrm{mm}$ umbilical trocar, and a $2-\mathrm{mm}$ camera was placed in the $2-\mathrm{mm}$ right trocar. This setting reduced the role of a 2-mm instrument; it pulled the peritoneum during stitching and held the thread rather than the needle during knotting. However, the poor image quality and limited visual field shown by the 2-mm camera should be technically improved.

\section{Operation time for unilateral hernia repair}

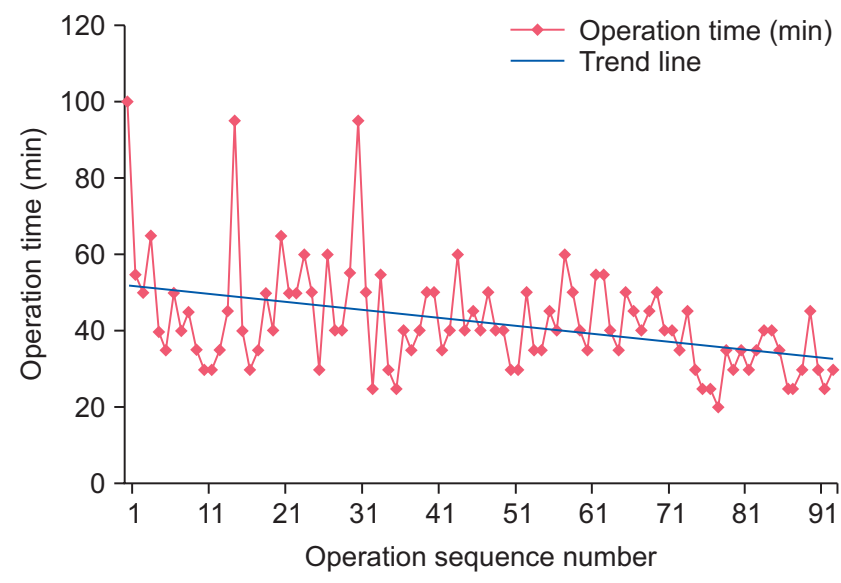

Fig. 5. Change in operating time for unilateral inguinal hernia repair in 93 patients. As surgical experiences were accumulated, the operating time tended to decrease gradually. 
In the literature, needlescopic instruments or puncture needles were mostly used in the extracorporeal technique. ${ }^{13-16}$ On the other hand, Schier reported a large series of patients who underwent a laparoscopic intracorporeal suture repair using 2-mm instruments. ${ }^{17}$ He closed the internal ring with a single $\mathrm{N}$-shaped suture instead of a purse-string suture. Lee et al. ${ }^{9}$ reported the intracorporeal purse-string suture technique using not only 2-mm needlescopic instruments but also 3-mm instruments. The mean operating time for laparoscopic repair ranged from 10 to 47.6 minutes for unilateral hernia and from 14 to 57.1 minutes for bilateral hernia. ${ }^{18}$ When compared with the intracorporeal purse suture technique, percutaneous extracorporeal closure was shown to have a significant reduction in operating time. ${ }^{9,13,16,17}$ In this study, the mean operating time was 42 minutes for unilateral repair and 46 minutes for bilateral repair. As surgical experiences were accumulated, the operating time gradually decreased (Fig. 5). We performed laparoscopic repair in small infants but experienced difficulties for intracorporeal suture due to a narrow working space, particularly if they are less than 6 months old or weighed less than $10 \mathrm{~kg}$. We performed 11 laparoscopic repairs for $8 \mathrm{pa}^{-}$ tients weighing less than $10 \mathrm{~kg}$, who had no recurrence. However, in such patients, further studies are needed to determine which surgery is better between open repair and laparoscopy.

The major concern about the laparoscopic approach is its higher recurrence rate than open repair, ranging from $0.8 \%$ to $4.3 \%$. Various causes for recurrence have been suggested, including tension at the closure of the internal inguinal ring, large hernias, technical problem with the suture, and the skip area over the vas deferens and spermatic vessels around the internal inguinal ring. ${ }^{3}$ Schier described that all recurrences occurred when the suture was placed too laterally because of the fear of injuring the epigastric vessels. ${ }^{17}$ This technical failure resulted in the recurrent internal opening observed between the epigastric vessels (medially) and former suture knot (laterally). As a solution, he suggested that the recurrence rate was reduced by including some tissue from the medial side of the epigastric vessels.

In this study, we experienced 3 recurrent hernias. One case previously had a large sliding hernia and was corrected with open repair, in which the former suture knot was found in the middle of the recurrent hernia sac. The other 2 cases were corrected using laparoscopic repair again, in which the former suture knot was observed at the orifice of the recanalized internal ring (Fig. 3). In these 2 cases, the cause of the recurrence was thought to be tissue tear as Schier described. ${ }^{17}$ When the suture is placed too superficially on some parts of the internal ring, the peritoneum is torn under pressure, and then the knot is located at the opponent side of the recurrent orifice. As mentioned earlier, when suturing the internal in- guinal ring, including some tissues while avoiding the injury of the vas deferens and vessels is important. For the epigastric vessels on the upper medial side, and the vas deferens and spermatic vessels on the lower side, sutures should be placed as closely as required to the structures and should not create skip areas. ${ }^{3,17}$ The upper lateral side of the internal ring has no structure to avoid the injury but is also important when suturing. As more underlying tissue (muscular tissue) is included and fixed at the upper lateral side, ${ }^{17}$ the medial loose umbilical fold moves laterally to allow for tension-free suture. At the final step, the scrotum should also be squeezed and pressed on the skin over the inguinal canal before tying the knot air-tightly. After stitching, these 2 procedures should be performed in harmony to completely obliterate the space of the hernia sac. In this study, we guessed that the low incidence of postoperative hydrocele was attributed to these procedures, although herniotomy was not performed in most patients.

In conclusion, laparoscopic intracorporeal purse-string suture using 2-mm instruments is safe and technically feasible for pediatric inguinal hernias, with excellent cosmetic results. Our laparoscopic approach showed comparable results of recurrence and complication rates without herniotomy. However, more experience is needed to reduce the recurrence rate and shorten the operation time.

\section{ORCID}

Jeong Min Lim, https://orcid.org/0000-0002-3243-4423

Hye Kyung Chang, https://orcid.org/0000-0003-2537-7464

Sun Jin Park, https://orcid.org/0000-0001-7117-4479

\section{AUTHORS' CONTRIBUTIONS}

Conceptualization: SJP. Formal analysis: JML. Methodology: JML, SJP. Writing-original draft: JML, SJP. Writingreview and editing: HKC, SJP.

\section{CONFLICT OF INTEREST}

None.

\section{FUNDING}

None.

\section{ACKNOWLEDGMENTS}

None. 


\section{REFERENCES}

1) Abd-Alrazek M, Alsherbiny H, Mahfouz M, et al. Laparoscopic pediatric inguinal hernia repair: a controlled randomized study. J Pediatr Surg 2017;52:1539-1544.

2) Schier F, Montupet P, Esposito C. Laparoscopic inguinal herniorrhaphy in children: a three-center experience with 933 repairs. J Pediatr Surg 2002;37:395-397.

3) Shalaby R, Ismail M, Samaha A, et al. Laparoscopic inguinal hernia repair; experience with 874 children. J Pediatr Surg 2014;49: 460-464.

4) McClain L, Streck C, Lesher A, Cina R, Hebra A. Laparoscopic needle-assisted inguinal hernia repair in 495 children. Surg Endosc 2015;29:781-786.

5) Lee SR, Choi SB. The efficacy of laparoscopic intracorporeal linear suture technique as a strategy for reducing recurrences in pediatric inguinal hernia. Hernia 2017;21:425-433.

6) Saranga Bharathi R, Arora M, Baskaran V. Pediatric inguinal hernia: laparoscopic versus open surgery. JSLS 2008;12:277-281.

7) Dreuning K, Maat S, Twisk J, van Heurn E, Derikx J. Laparoscopic versus open pediatric inguinal hernia repair: state-of-theart comparison and future perspectives from a meta-analysis. Surg Endosc 2019;33:3177-3191.

8) Ostlie DJ, Ponsky TA. Technical options of the laparoscopic pediatric inguinal hernia repair. J Laparoendosc Adv Surg Tech A 2014;24:194-198.

9) Lee DY, Baik YH, Kwak BS, Oh MG, Choi WY. A purse-string suture at the level of internal inguinal ring, taking only the peritoneum leaving the distal sac: is it enough for inguinal hernia in pediatric patients? Hernia 2015;19:607-610.

10) Schier F. Laparoscopic herniorrhaphy in girls. J Pediatr Surg 1998; 33:1495-1497.

11) Marte A, Sabatino MD, Borrelli M, Parmeggiani P. Decreased recurrence rate in the laparoscopic herniorraphy in children: comparison between two techniques. J Laparoendosc Adv Surg Tech A 2009;19:259-262.

12) Yip KF, Tam PK, Li MK. Laparoscopic flip-flap hernioplasty: an innovative technique for pediatric hernia surgery. Surg Endosc 2004;18:1126-1129.

13) Shalaby R, Elsaied A, Shehata S, et al. Needlescopic assisted internal ring suturing; a novel application of low-cost home-made instruments for pediatric inguinal hernia repair. Hernia 2019;23: 1279-1289.

14) Shalaby RY, Fawy M, Soliman SM, Dorgham A. A new simplified technique for needlescopic inguinal herniorrhaphy in children. J Pediatr Surg 2006;41:863-867.

15) Shalaby R, Ismail M, Dorgham A, et al. Laparoscopic hernia repair in infancy and childhood: evaluation of 2 different techniques. J Pediatr Surg 2010;45:2210-2216.

16) Wang YJ, Zhang QL, Chen L, et al. Laparoscopic Percutaneous Extraperitoneal Internal Ring Closure for Pediatric Inguinal Hernia: 1,142 Cases. J Laparoendosc Adv Surg Tech A 2019;29:845851.

17) Schier F. Laparoscopic inguinal hernia repair-a prospective personal series of 542 children. J Pediatr Surg 2006;41:1081-1084.

18) Esposito C, Escolino M, Turra F, et al. Current concepts in the management of inguinal hernia and hydrocele in pediatric patients in laparoscopic era. Semin Pediatr Surg 2016;25:232-240. 